\title{
Hadron multiplicities at the HERMES experiment
}

\section{Gevorg Karyan*t}

A. I. Alikhanyan National Science Laboratory, Yerevan, Armenia

E-mail: gevkar@mail.desy.de

The HERMES collaboration has measured charge-separated pion and kaon multiplicities in semiinclusive deep-inelastic scattering using $27.6 \mathrm{GeV}$ electron or positron beam scattering off a hydrogen or deuterium target. The results are presented as functions of the Bjorken variable $x$, the negative squared four-momentum transfer $Q^{2}$, a hadron fractional energy $z$ and the hadron's transverse momentum. These data will be very useful to understand the quark-fragmentation process in deep-inelastic hadron electro-production and will serve as crucial input in the understanding of charge/flavour separated fragmentation functions. Furthermore, it provides important information on the transverse-momentum dependence of hadron production.

XXI International Workshop on Deep-Inelastic Scattering and Related Subjects,

22-26 April 2013

Marseilles, France

* Speaker.

${ }^{\dagger}$ for the HERMES Collaboration 


\section{Introduction}

The measurement of the hadron multiplicities is one of the key tools to study the hadronization process. The lack of a complete theory to describe a hadron production process leads to a phenomenological understanding of a quark fragmentation into hadrons. According to the factorization theorem[1] the cross section of a hadron production in semi-inclusive deep inelastic leptonnucleon scattering (SIDIS) process is described by parton distribution functions and fragmentation functions. On the other hand the universality for the fragmentation functions[2] gives a good chance to compare those with fragmentation functions extracted from different kind of precesses and consider them as a global input for the further studies. Understanding the hadronization process is an essential element of a complete picture of the interaction of quarks in Quantum ChromoDynamics (QCD), and is basic for the understanding of the dynamics of quark-quark, gluon-gluon, and quark-gluon interactions.

\section{Data Extraction}

The data were collected with the HERMES spectrometer using $27.6 \mathrm{GeV}$ electron or positron beams stored in HERA at DESY[3]. To select deep-inelastic scattered (DIS) lepton the following

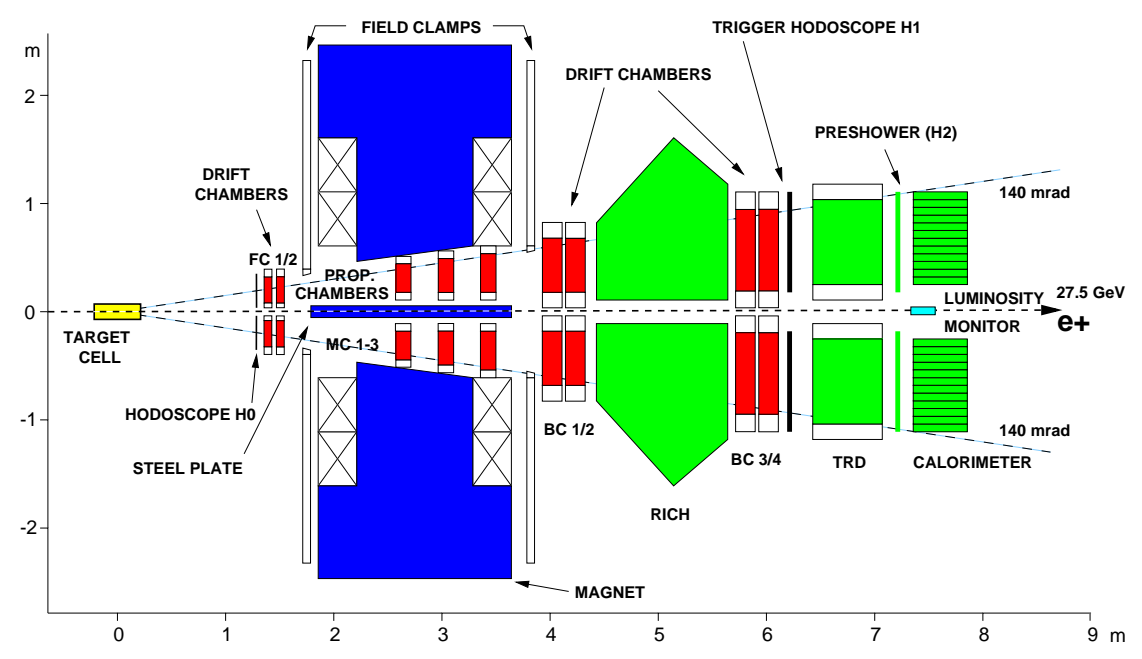

Figure 1: The HERMES spectrometer.

requirements were used for it's kinematics: $Q^{2}>1 \mathrm{GeV}^{2}, W^{2}>10 \mathrm{GeV}^{2}$ and $0.1<y=v / E<0.85$, where $W$ is the invariant mass of virtual photon-nucleon system and $E$ is the beam energy. The cut on the invariant mass imposed to suppress the resonance contribution into data and constrain on $y$ limits the magnitude of radiative corrections.

For charged hadron identification, dual-radiator ring-imaging Čerenkov detector (RICH) was used[4], which allows to identify pions, kaons and (anti)proton. They were selected within the following kinematical conditions: $2 \mathrm{GeV}<p_{h}<15 \mathrm{GeV}$, where $p_{h}$ is the hadron momentum and the cut on $z$ is applied $0.2<z<0.8$ to exclude the region at high $z$, which is dominated by exclusive processes, and to suppress he target fragmentation region at low $z$. The experimental observable is the hadron 
multiplicity which is the hadron yield $N^{h}$ normalized to the $N_{D I S}$ (DIS yield) :

$$
M^{h}\left(x_{B}, Q^{2}, z, P_{h \perp}\right)=\frac{1}{\frac{d^{2} N_{D I S}\left(x_{B}, Q^{2}\right)}{d x_{B} d Q^{2}}} \cdot \int_{0}^{2 \pi} \frac{d^{5} N^{h}\left(x_{B}, Q^{2}, z, P_{h \perp}, \phi_{h}\right)}{d x_{B} d Q^{2} d z d P_{h \perp} d \phi_{h}} d \phi_{h}
$$

At Born-level, the multiplicities, i.e. the multiplicities with no limitations in geometric acceptance and corrected for radiative effects and detector resolution, are extracted from measured multiplicities binned in 3 dimensions: $\left(x_{B}, z, P_{h \perp}\right)$ when the multiplicities as a function of the $x_{B}$ are desired, and $\left(Q^{2}, z, P_{h \perp}\right)$ when they have been a function of the $Q^{2}$. Due to the strong correlation between the $x_{B}$ and the $Q^{2}$ in the HERMES kinematical acceptance, simultaneous data binning in these two variables would not have an impact on the extraction. A $\phi_{h}$ binning has been omitted because of limited statistical precision. However, a possible impact of the $\phi_{h}$ dependence of the unpolarized semi-inclusive cross section and its acceptance have been accounted for in the systematic uncertainties.

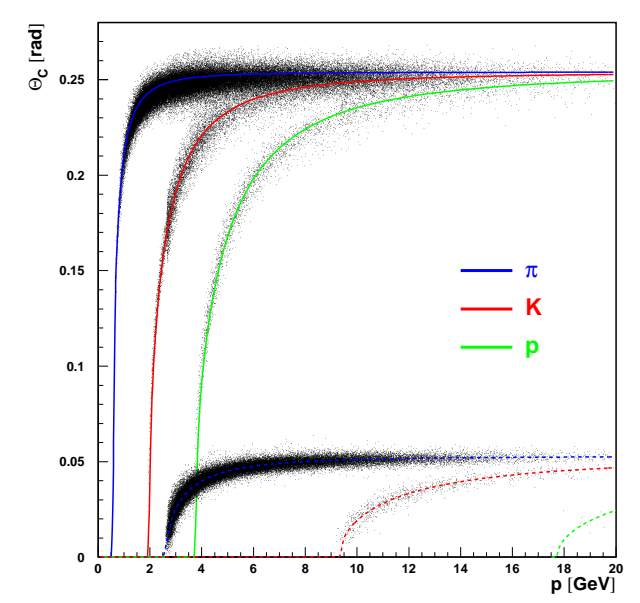

Figure 2: Momentum dependence of the Čerenkov angle for different hadron types and radiators. The upper band corresponds to the aerogel and the lower band to the $\mathrm{C}_{4} \mathrm{~F}_{10}$ gas respectively.

\section{Results}

The Born multiplicities, after correction for the exclusive vector-meson production, are presented in Fig. 3. Here they are shown for both proton and deuteron targets as a function of the energy fraction $z$ in four panels corresponding to different type of final-state hadron. The individual panels compare data for a given hadron type taken with a proton (full circles) or deuteron (empty squares) target. The multiplicity ratio of $\pi^{+}$to $\pi^{-}$for the proton (deuteron) ranges from 1.2 (1.1) in the first $z$ bin to 2.6 (1.8) in the last $z$ bin. These results can be attributed to the dominance of scattering off the $u$ quarks, reflecting the fact that the fragmentation process $u \rightarrow \pi^{+}$is favored, while the process $u \rightarrow \pi^{-}$is unfavored. With rising $z$, this effect is enhanced. For kaons, the $K^{+}$multiplicity for the proton is slightly larger than for the deuteron, while within errors for $K^{-}$the multiplicities are equal. The ratio $K^{+} / K^{-}$rises from 1.5 to 5.7 (proton target) and from 1.3 to 4.6 (deuteron target) for $0.2<z<0.8$, reflecting the fact that $K^{-}$cannot be produced through 


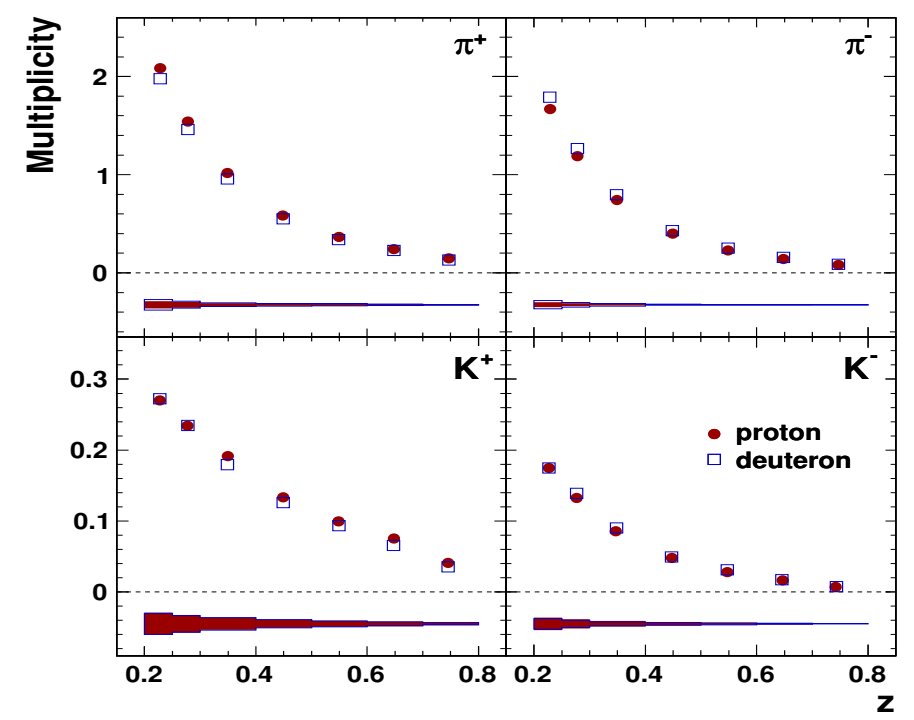

Figure 3: Multiplicities corrected for exclusive vector mesons as a function of $z$ from a proton target (full circles) and a deuteron target (empty squares). The error bars for the statistical uncertainties are too small to be visible. The systematic uncertainties are given by the error bands.

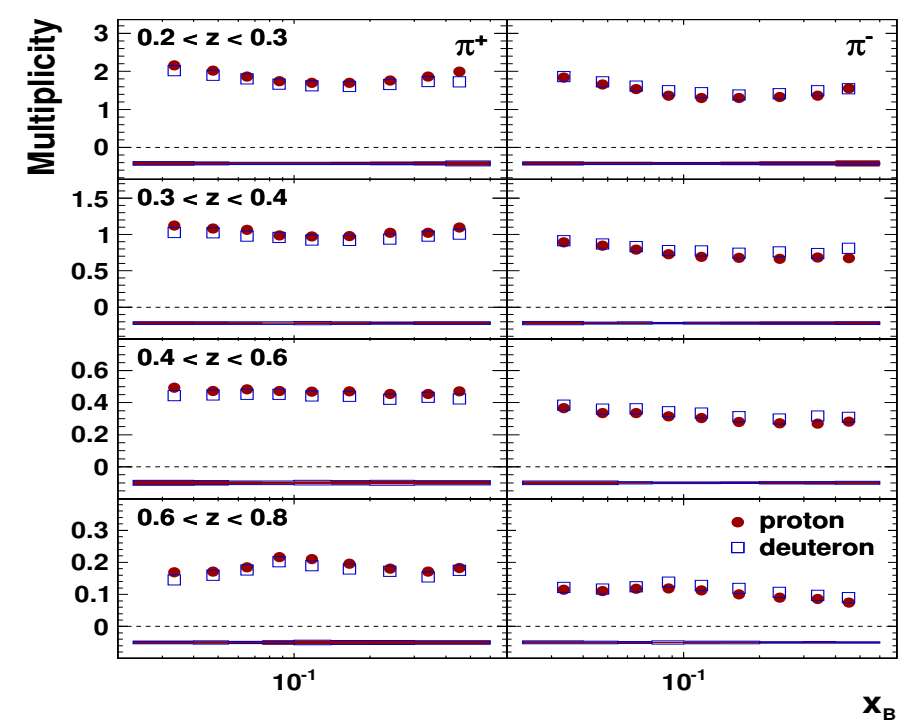

Figure 4: Multiplicities of charged pions for the proton (full circles) and the deuteron (empty squares) as a function of $x_{B}$ in four $z$ bins. Positive charge is on the left and negative charge is on the right of the panel. Uncertainties are as in Fig. 3.

favored fragmentation from the nucleon valence quarks. In Figs. 2-3 the multiplicities are presented for positively and negatively charged pions and kaons as a function of the Bjorken $x_{B}$. The dependencies on transverse momentum of a hadron are shown in Fig. 4. The features of the $P_{h \perp}$ distributions result from the combined effects of the initial transverse motion of the struck quark 


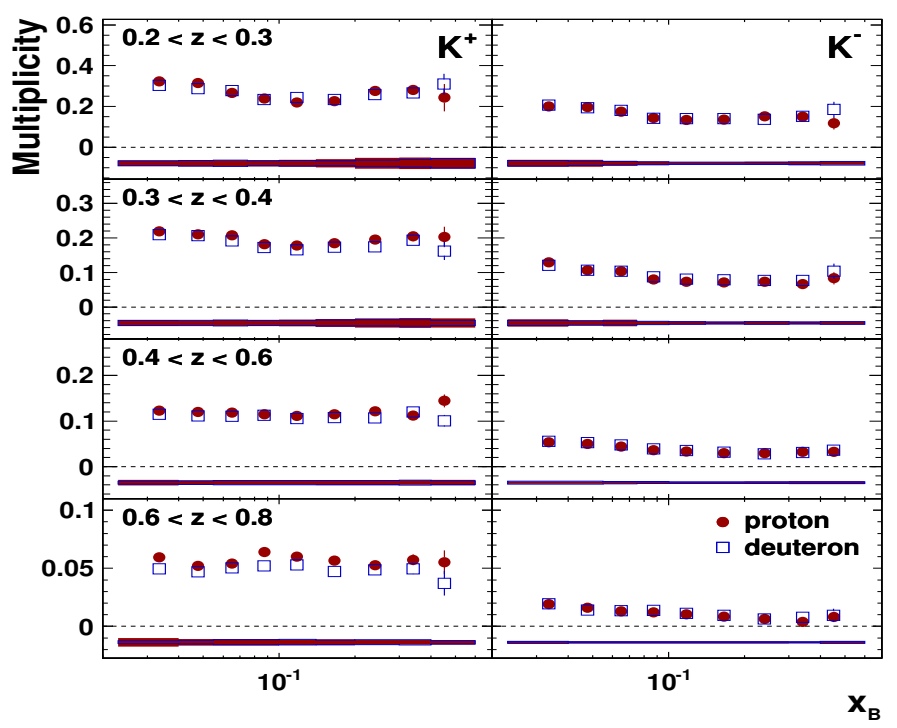

Figure 5: Multiplicities of charged kaons for the proton (full circles) and the deuteron (empty squares) as a function of $x_{B}$ in four $z$ bins. Positive charge is on the left and negative charge is on the right of the panel. Uncertainties are as in Fig. 3.

in SIDIS, of its emission of soft gluon radiation, and of the component of transverse momentum which is generated by the fragmentation process.
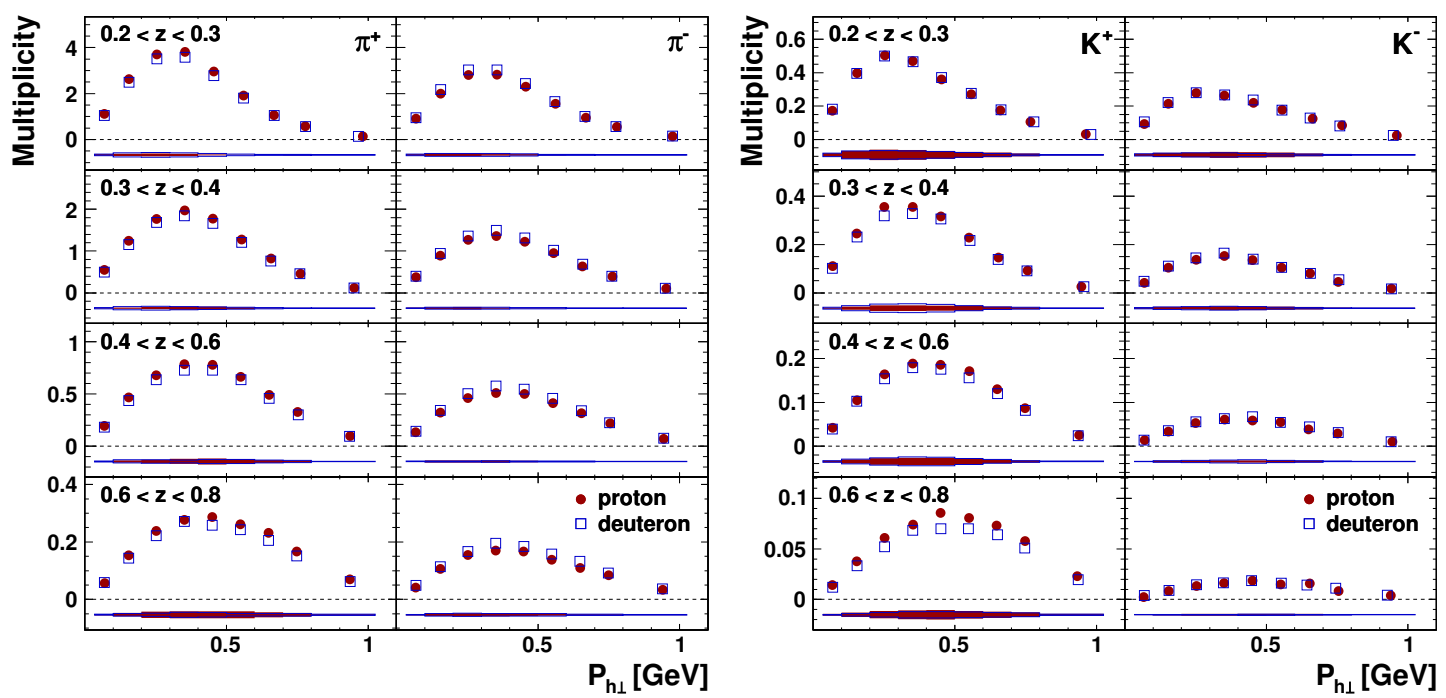

Figure 6: Multiplicities of charged pions and kaons for the proton (full circles) and the deuteron (empty squares) as a function of $P_{h \perp}$ in four $z$ bins. Positive charges are on the left and negative charges are on the right of each panel. Uncertainties are as in Fig. 3. 


\section{Conclusions}

The high statistical data set for charged pion $\left(\pi^{+}, \pi^{-}\right)$and kaon $\left(K^{+}, K^{-}\right)$multiplicities have been presented for proton and deuteron targets[5]. The data shows that the fragmentation is favored for the hadrons containing the struck quark as a valence quark. The dependence of multiplicities on $P_{h \perp}$ can be used to provide constraints on the models of the fragmenation process. These high statistical data will allow more reliable extraction of unfavored fragmentation functions and also the strange quark fragmentation functions which current knowledge is not yet well developed.

\section{References}

[1] J. C. Collins, L. Frankfurt, and M. Strikman, Phys. Rev. D 56, 2982âĂŞ3006 (1997).

[2] S. Albino, B. A. Kniehl, G. Kramer, and C. Sandoval, Phys. Rev. D75, 034018 (2007).

[3] K. Akertaff et al., HERMES coll., Nucl. Instrum. Methods A417 239 (1998).

[4] N. Akopov et al. NIM A479 (2002) 511.

[5] A. Airapetian et al. Phys. Rev D87, 074029 (2013). 